\title{
The Importance of Home Versus 24-Hour Ambulatory Blood Pressure Monitoring and Assessment of Blood Pressure Variability in Hypertension
}

\author{
Magdás Annamária*, Belényi Boglárka, Gaburoi Adina, Incze Alexandru \\ University of Medicine and Pharmacy Tirgu Mures, Romania
}

Background: A number of studies reveal that home blood pressure variability is associated with cardiovascular risk factors. However, we do not have a consensus regarding the variability index and the frequency of measurements.

Objective: The aim of the study was to assess home blood pressure variability for a period of 7 consecutive days and 24-hour ambulatory blood pressure variability using the average real variability index and to test whether home blood pressure variability represents a suitable parameter for long-term monitoring of the hypertensive patients. Material and methods: A number of 31 hypertensive patients were included in the study, 8 male, 23 female, mean age 60.19 7.35 years. At the inclusion ambulatory blood pressure monitoring was performed, home blood pressure monitoring was carried out for 7 consecutive days with 2 measurements daily. We compared ambulatory blood pressure values, variability using paired t-test. We were looking for correlations between HBP values and cardiovascular risk factors. Results: Ambulatory versus home blood pressure derived mean blood pressure was $131.38 \pm 15.2$ versus $131.93 \pm 8.25, p=0.81$. Ambulatory derived variability was $10.65 \pm 2.05$ versus home variability $10.56 \pm 4.83, p=0.91$. Home versus ambulatory pulse pressure was $51.8 \pm 9.06 \mathrm{mmHg}$ vs. $54.9 \pm 11.9$ $\mathrm{mmHg}, \mathrm{p}=0.046$. We found positive correlation between HBPV and home BP values, $\mathrm{p}=0.027, \mathrm{r} 2=0.1577$, (Cl: 0.04967 to 0.6588). Home, as well as ambulatory derived variability were positively correlated to age $\mathrm{p}=0.043, \mathrm{r} 2=0.1377$ (Cl: 0.01234 to 0.6451$)$ versus $p<0.0001, \mathrm{Cl}$ : 0.3870 to $0.8220, r 2=0.4302$. Conclusion: Assessment of home blood pressure monitoring and variability could represent a well-tolerated alternative for long-term follow-up of hypertension management.

Keywords: ambulatory blood pressure, home blood pressure variability, hypertension

Received: 29 November 2015 / Accepted: 20 March 2016

\section{Introduction}

The degree of blood pressure control is well highlighted by the SEPHAR II study, in which $59.1 \%$ of the hypertensive patients are treated and blood pressure control was recorded in only $25 \%$ of them [1]. Therefore, to increase treatment response and patient compliance, implementation of self-monitoring have to be encouraged. Although the 24-hour ambulatory monitoring of blood pressure represents the gold standard for the diagnosis and evaluation of treatment response in hypertension, home blood pressure measurement is widely available and well tolerated by the patient $[2,3]$. Accumulating evidence reveals that an increase in 24-hour blood pressure variability as well as in home blood pressure variability is associated with end-organ damage [4-7]. The self-measurement of blood pressure at home offers the possibility for a long-term monitoring of the blood pressure variability and removes the whitecoat phenomenon. It has been described that an increase in home blood pressure variability independent of mean blood pressure value, has a predictive value in the development of cardiac, vascular and renal damage [8]. Until now, there is a great diversity among studies regarding protocols including duration of monitoring, number of measure-

* Correspondence to: Annamária Magdás

E-mail: annamaria.magdas@umftgm.ro ments as well as the use of a reliable parameter to assess home blood pressure variability [9]. Despite of available data regarding the relationship of ambulatory and home blood pressure variability with end-organ damage and cardiovascular mortality, there is a lack of studies comparing the utility of home versus ambulatory variability of blood pressure. Therefore, the aim of the present study was to assess home blood pressure variability for a period of 7 consecutive days and 24-hour ambulatory blood pressure variability, using the average real variability index and to test whether home blood pressure variability represents a suitable parameter for long-term follow-up of the hypertensive patients.

\section{Methods}

This observational study was performed in County Clinical Hospital Targu-Mures, department for Internal Medicine IV and included 31 hypertensive patients, 8 males and 23 females, mean age was $60.19 \pm 7.35$ years. All subjects gave written informed consent and the study was approved by Local Ethical Committee according to the International Ethical Guidelines and Declaration of Helsinki. Inclusion criteria were: history of hypertension, use of antihypertensive medication. Exclusion criteria were: patients with arrhythmias, congestive heart failure NYHA class III/IV, coagulation disease, non-cooperative patients. 
Twenty four-hour ambulatory BP monitoring was performed with a validated device (ABPM $05^{\circ}$, Meditech Ltd., Hungary) applied on the non-dominant arm of the patients. Measurements started between 8-10 am, the frequency of the blood pressure measurements was set at 20 minutes daytime and nighttime. After 24-hour blood pressure recording, home blood pressure monitoring was performed with validated semi-automated oscillometric devices according to ESC practical aspects and ESH practical guidelines on home blood pressure monitoring. Patients were trained how to perform correct measurements. A 24 $\mathrm{cm}$ standard cuff was placed approximately $2-3 \mathrm{~cm}$ above the bend of the elbow. Duplicate measurements were performed, each about 1-2 minutes apart, in the morning before drug intake and evening before meal, for a period of 7 consecutive days, prior to the one month visit. Measurements were performed in a quiet room with the patients seated comfortably for at least 5 minutes with the arm resting on a table and the cuff at heart level $[10,11]$. The results were noted in a logbook after each measurement. Average blood pressure was calculated after excluding the values of the first monitoring day. Ambulatory as well as home blood pressure variability were calculated according to the formula of average real variability (ARV) using a computerized program [12]. Ambulatory blood pressure data like mean daytime, nighttime and 24-hour BP, pulse pressure, morning surge, diurnal/nocturnal index were obtained automatically from the measurement device. Home monitoring derived mean systolic/diastolic blood pressure, home variability and pulse pressure were calculated in MS Excel program. Demographic characteristics (age, gender, high, weight), the treatment regimen of each patient were collected in a questionnaire. Data were collected as raw data, using MS Excel program, statistical analysis were performed using Graph Pad Prism version 5 statistical software. Numerical data are represented as mean \pm SD. Means were compared using paired $t$-test, correlations were calculated with Pearson's correlation test for data representing Gaussian distribution. A p value $\leq 0.05$ was considered statistically significant with a confidence interval set at $95 \%$.

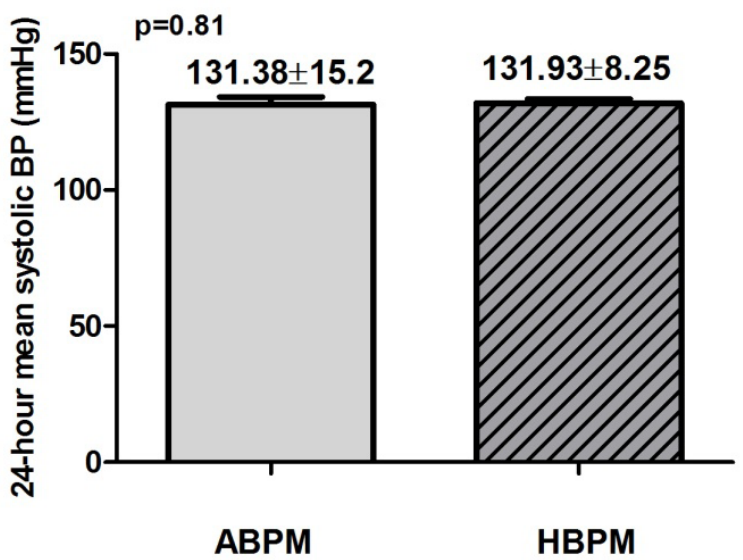

Fig. 1. Difference in ABPM versus HBPM derived mean systolic BP

\section{Results}

A number of 31 treated hypertensive patients were included in the study, 8 male and 23 female, mean age was $60.19 \pm 7.35$ years. Body mass index was $30 \pm 2.46 \mathrm{~kg} / \mathrm{m}^{2}$, there were 11 dippers and 20 non-dippers. Regarding laboratory parameters: mean cholesterol level was $204.4 \pm 24.7$ $\mathrm{mg} / \mathrm{dl}$, triglycerides were $186.1 \pm 78.9$. Mean systolic blood pressure measured by ambulatory and home monitoring were almost equal (figure 1). Blood pressure variability determined by average real variability showed no significant difference at ABPM versus HBPM (figure 2). Ambulatory monitoring derived diastolic blood pressure was statistically significant lower compared to home monitoring diastolic blood pressure, $76.6 \pm 6.2 \mathrm{mmHg}$ versus $83.7 \pm 6$ $\mathrm{mmHg}, \mathrm{p}<0.0001$. Ambulatory monitoring derived pulse pressure was significantly higher compared to home monitoring derived pulse pressure, $54.9 \pm 11.9$ versus $51.8 \pm 9.06$, $\mathrm{p}=0.046$. Home and ambulatory blood pressure variability were positively correlated to age $\mathrm{p}=0.043, \mathrm{r} 2=0.1377$ (CI: 0.01234 to 0.6451 ) versus $\mathrm{p}<0.0001, \mathrm{CI}: 0.3870$ to $0.8220, \mathrm{r} 2=0.4302$.

We found positive correlation between 24-hour systolic blood pressure and its variability defined with average real variability, $\mathrm{p}=0.013, \mathrm{r}^{2}=0.2001, \mathrm{CI}: 0.1037$ to 0.6956 . This correlation was observed also between home measured systolic blood pressure values and blood pressure variability calculated for at least 14 measurements, $\mathrm{p}=0.027$, $\mathrm{r}^{2}=0.1577$, CI: 0.04967 to 0.6588 (figure 3 ). Home variability was positively correlated to home pulse pressure, $\mathrm{p}<0.0001, \mathrm{r}^{2}=0.4102$, CI: 0.3700 to 0.8108 .

\section{Discussion}

An increasing number of studies investigating the relationship between home blood pressure variability with cardiovascular risk factors found that female gender, advanced age, increased mean blood pressure, smoking as well as other factors are associated with increased HBPV $[13,14]$. Evidence is available that an increased day-by-day variability assessed by home monitoring, independently of average home blood pressure levels, is a predictor of development

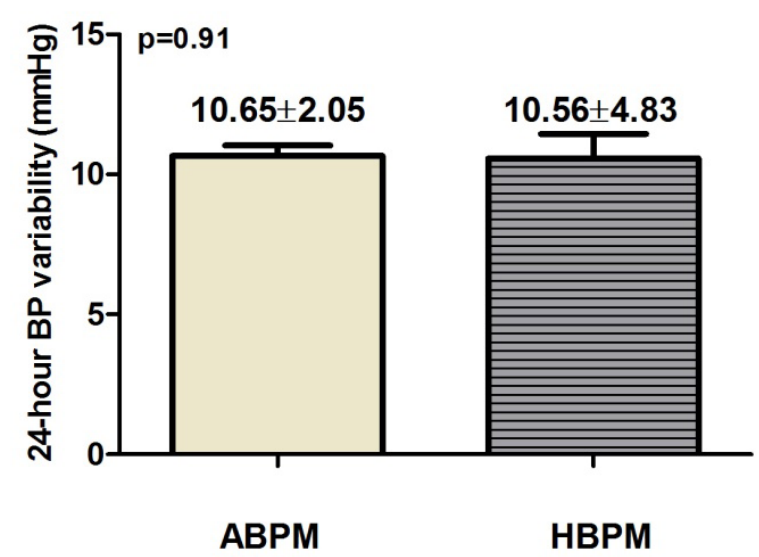

Fig. 2. Difference in BP variability defined as average real variability 
and progression of end-organ damage [15]. In the FinnHome study, increased home blood pressure variability performed over seven consecutive days was associated with a higher risk of cardiovascular events after a period of 7.8 years of follow-up [16]. In contrast to the mentioned study, we compared ambulatory and home blood pressure values as well as variability and investigated the relationship between blood pressure monitoring derived parameters and variability in a high-risk population defined by elevated total serum cholesterol and triglyceride levels as well as age.

We found that ambulatory and home monitoring derived mean systolic blood pressure and variability defined as average real variability were almost equal. Ambulatory monitoring derived mean diastolic blood pressure was greater than the home measured. Therefore, ambulatory pulse pressure was higher than home monitoring derived pulse pressure. A possible explanation could be that blood pressure fluctuation is a complex phenomenon influenced by arterial compliance, humoral factors, and behavioral changes [17]. Ambulatory as well as home monitoring derived variability defined as average real variability, showed positive correlation with mean systolic blood pressure levels. It is known, that ambulatory blood pressure variability correlates with arterial stiffness defined by pulse pressure [18]. Based on our findings, this observation is also available for home blood pressure variability which showed positive correlation with home measured pulse pressure.

Self-measurement of blood pressure is a comfortable and cheap method and it represents an appropriate method for long-term follow-up of treated patients, but ABPM and HBPM provide complementary information, therefore in clinical practice, the appropriate combination of this methods is required [19].

Therefore, home blood pressure monitoring performed for at least 7 consecutive days, prior to programmed doctor visit and the assessment of home variability using the average real variability formula, could represent a welltolerated and cost-efficient method in terms of long-term follow-up of the hypertensive patient [20].

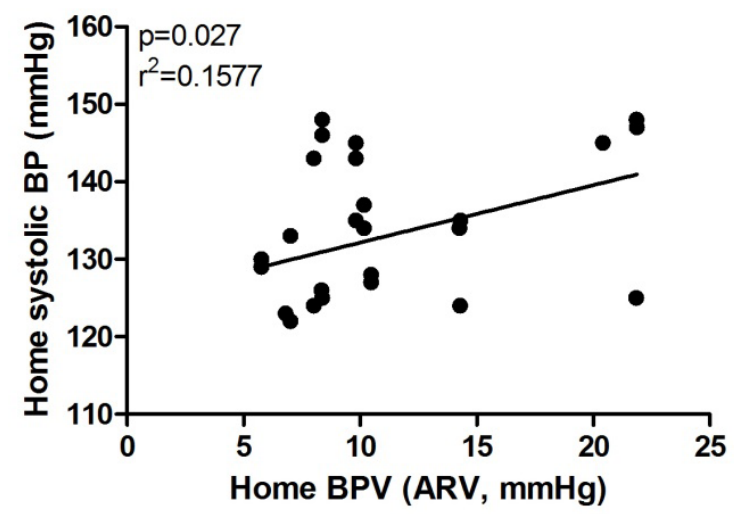

Fig. 3. Pearson's correlation for HBPV and home sytolic BP

\section{Conclusion}

Although the 24-hour blood pressure monitoring remains the gold standard in terms of long-term follow-up of hypertension management, self-measurement of the blood pressure and calculation of its variability represents a complementary method for long-term follow-up, and is accepted by the patients. In treated hypertensive patients with optimal controlled blood pressure values at ambulatory monitoring, if at one month follow-up, home variability values are close to the ambulatory ARV values, it might be used as a measure of visit-to-visit blood pressure control, below obtaining optimal blood pressure values, but future studies are needed to define a universally accepted index and an optimal self-monitoring schedule.

\section{Acknowledgement}

The financial support for this study was offered by the Internal Research Grant of the University of Medicine and Pharmacy Targu-Mures, contract number 16/23.12.2014.

\section{Conflict of interest}

None to declare.

\section{References}

1. Dorobanțu M, Darabont R, Ghiorghe S, et al. Profile of the Romanian hypertensive patient data from SEPHAR II study. Rom J Intern Med. 2012;50(4):285-96.

2. Hermida RC, Smolensky MH, Ayala DE, et al. Ambulatory blood pressure monitoring recommendations for the diagnosis of adult hypertension, assessment of cardiovascular and other hypertension-associated risk, and attainment of therapeutic goals. Chronobiol Int 2013;30(3):3.

3. Parati G, Stergiou GS, Asmar R, et al. European Society of Hypertension Practice Guidelines for home blood pressure monitoring. J of Human Hypertens 2010;24:779-785.

4. Kikuya M, Ohkubo T, Metoki $\mathrm{H}$, et al. Day-by-day variability of blood pressure and heart rate at home as a novel predictor of prognosis: the Ohasama study. Hypertens 2008;52:1045-1050.

5. Ishikura K, Obara T, Kato T, et al. Associations between day-by-day variability in blood pressure measured at home and antihypertensive drugs: the J-HOME- morning study. Clin Exp Hypertens 2012;34(4):297304 .

6. Hansen TW, ThijsL, LiY et al. Prognostic Value of Reading-to-Reading Blood Pressure Variability Over 24 Hours in 8938 Subjects From 11 Populations. Hypertens 2010;55:1049-1057.

7. Johansson JK, Niranen TJ, Puukka PJ, et al. Factors affecting the variability of home-measured blood pressure and heart rate: the Finnhome study. J Hypertens 2010;28(9):1836-1845

8. Ushigome E, Fukui M, Hamaguchi M, et al. The coefficient variation of home blood pressure is a novel factor associated with macroalbuminuria in type 2 diabetes mellitus. Hypertens Res 2011;34:1271-1275

9. Parati G, Ochoa JE, Lombardi $\mathrm{C}$ et al. Blood pressure variability: Assessment, predictive value, and potential as a therapeutic target. Curr Hypertens Rep 2015;17-23

10. Singh V. Home blood pressure monitoring, practical aspects - second in series. E journal of Cardiology Practice 2015;13:13

11. Mancia G, Fagard R, Narkiewicz K, et al. The Task Force for the management of arterial hypertension of the European Society of Hypertension (ESH) and of the European Society of Cardiology (ESC). J Hypertens 2013;31:1281-1357

12. Mena. L, Pintos S, Queipo NV, et al. A reliable index for the prognostic significance of blood pressure variability. J Hypertens 2005;23(3):505511

13. Asayama K, Kikuya M, Schutte R et al. Home blood pressure variability as cardiovascular risk factor in the population of Ohasama. Hypertens 2013;61:61-69

14. Fukui M, Ushigome E, Tanaka M et al. Home blood pressure variability on one occasion is a novel factor associate with arterial stiffness in patients with type 2 diabetes. Hypertens Res 2013;36:219-225 
15. Matsui $\mathrm{Y}$, Ishikawa J, Eguchi K, et al. Maximum value of home blood pressure: a novel indicator of target organ damage in hypertension. Hypertens 2011;57(6):1087-93

16. Johansson JK, Niiranen TJ, Puukka PJ, Jula AM. Prognostic value of the variability in home-measured blood pressure and heart rate: the FinnHome Study. Hypertens 2012;59(2):212-218

17. Parati G, Juan EO, Lombardi C, Bilo G. Assessment and management of blood-pressure variability. Nat Rev Cardiol 2013;10:143-155

18. Fujita H, Matsuoka S, Awazu M. White-coat andr white-coat effects correlate with 24-h pulse pressure and systolic blood pressure variability in children and young adults. Pediatr Cardiol. 2016;37(2):345-352

19. Paratia G, Stergiou G, , O'Brien E et al. European Society of Hypertension practice guidelines for ambulatory blood pressure monitoring. J Hypertens 2014;32:1359-1366

20. Parati G, Stergiou GS, Asmar R, et al. European Society of Hypertension Practice Guidelines for home blood pressure monitoring. Journal of Human Hypertension 2010;1-7 